\title{
Comment on “Dose Mapping Using Multipole Moments"
}

\author{
Elassaad Jemii \\ Physics Department, Faculty of Sciences Al-Zulfi, Majmaah University, Majmaah, Saudi Arabia \\ Email: elassaad_fsm@yahoo.fr
}

How to cite this paper: Jemii, E. (2017) Comment on "Dose Mapping Using Multipole Moments". Open Access Library Journal, 4: e3795.

https://doi.org/10.4236/oalib.1103795

Received: July 1, 2017

Accepted: August 7, 2017

Published: August 10, 2017

Copyright $\odot 2017$ by author and Open Access Library Inc.

This work is licensed under the Creative Commons Attribution International License (CC BY 4.0).

http://creativecommons.org/licenses/by/4.0/

\begin{abstract}
In the present work, we present a brief comment concerning the paper published by Loussaief et al. 2007 (Nucl. Inst. and Meth in Phys. Resh. A 580. 2007). The reference has used the multipole expansion method to determine the photon flux and the dose distribution in the vicinity of the Tunisian gamma irradiation facility. We have shown that the multipole expansion is useless and wrong for the calculation of the photon flux and the dose distribution in the vicinity of the CNSTN irradiator. We have also determined the dose distribution in the vicinity of the same irradiator, using a straightforward calculation, in order to show that we can abandon the multipole expansion method I our case.
\end{abstract}

\section{Subject Areas}

Nuclear Physics

\section{Keywords}

Multipole Expansion, Photon Flux Calculation, Dose Mapping, Gamma Source

\section{Introduction}

The ${ }^{60} \mathrm{Co}$ gamma irradiator, installed in the Center National for Nuclear Sciences and Technology (CNSTN), is used especially for irradiation purposes. It is designed for sterilization of medical devices and foodstuff irradiation. It is an extended source composed by eight pencils of $45 \mathrm{~cm}$ length arranged in two levels. Four pencils are mounted around a $7-\mathrm{cm}$ radius circle, upward along the $\mathrm{z}$-axis, whereas, the other four are mounted around a $4-\mathrm{cm}$ radius circle, downward the $\mathrm{z}$-axis [1]. 
Finally, the overall high of the source is equal to $90 \mathrm{~cm}$ and the mean radial extension is about $5.5 \mathrm{~cm}$. In a previous work [1], we have shown that, the extended source of the CNSTN is equivalent, less than one percent, to a pencil-like gamma source with the same activity and the same height. Using this previous result, we have shown determine the isodose curves in the vicinity of the CNSTN Irradiator.

\section{Multipole Expansion}

In order to calculate the photon flux and the dose rate in the vicinity of the ${ }^{60} \mathrm{Co}$ gamma source presented below, the author has used the multipole expansion as function of $r^{\prime} / r$, where $r^{\prime}$ is the vector position of an elementary source activity and $r$ is the vector position of the dosimeter relative to the source [2]. In its work [3], the author has calculated the gamma flux rates in 36 points in the vicinity of the source. The distance between the source and the plane of measurement points starts from $40 \mathrm{~cm}$ with a $25-\mathrm{cm}$ step along each direction, which forms a square mesh of $25 \mathrm{~cm}$ of length. Unfortunately, the author claims that, he has found a good agreement between the relative calculated values and the measurements.

Note that the multipole expansion, is valid only, if the distance between the source and the point of calculation the photon flux is very large compared to the source dimensions (in other word, the point is far away from the source). Given that, in some positions used by this reference, the ratio $r^{\prime} / r$ can be even bigger than one $\left(r=40 \mathrm{~cm}\right.$ and $\left.r^{\prime}=45 \mathrm{~cm}: r^{\prime} / r=1.125\right)$. Then a multipole expansion as a function of $r^{\prime} / r$, which can never converge, is wrong.

In fact, we can use the multipole expansion to calculate the photon flux, but it must be a development in terms of $\rho / r$ which is always very small, $\rho$ being the mean radial extension of the CNSTN source $(\rho \approx 5.5 \mathrm{~cm})$. Secondly, since we have shown that the CNSTN source was equivalent to a pencil like source, it is easier to determine the dose distribution using the straightforward expression than the multipole expansion.

The expression of the dose rate, in the vicinity of the CNSTN Irradiator, obtained in reference [4] is given by:

$$
d(X, Z)=K \frac{1}{X}\left(\operatorname{arctg}\left(\frac{Z+L}{X}\right)-\operatorname{arctg}\left(\frac{Z-L}{X}\right)\right) f(X, Z),
$$

where: $K$ is a constant.

Figure 1 shows the isodose curves $(d(X, Z)=$ constant $)$ in seven different plans parallel to the source $(X=40 \mathrm{~cm}$ to $X=190 \mathrm{~cm})$. Figure 1 also shows an absolute dose rate $(G y / h)$.

\section{Conclusion}

In the present, we have shown that the multipole expansion as function of $r^{\prime} / r$ cannot be used to determine the photon flux as well as the dose distribution in the vicinity of the CNSTN irradiator. In fact, if it is necessary to use such 


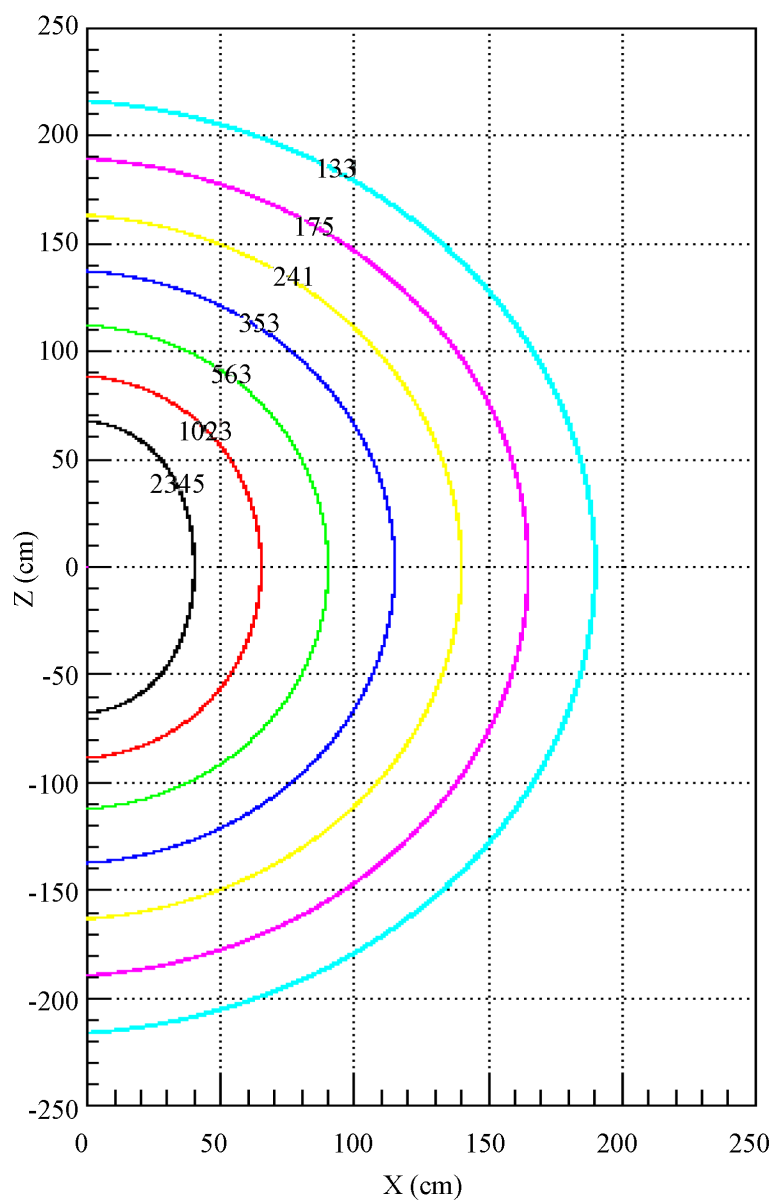

Figure 1. Isodose curves at seven vertical plans $(\mathrm{Gy} / \mathrm{h})$.

development, it will be as function of $\rho / r$, which is always very small. We have also shown that the dose mapping in the vicinity of the CNSTN Irradiator can be determined using a simple expression.

\section{References}

[1] Jemii, E., Mazouz, M., Ben Fredj, A. and Ghedira, L. (2011) Modeling of the Tunisian ${ }^{60} \mathrm{Co}$ Gamma Irradiator by a Coaxial Equal Height and Equal Activity Single Pencil. Radiation Physics and Chemistry, 80, 1158-1161.

https://doi.org/10.1016/j.radphyschem.2011.05.003

[2] Loussaief, A., Trabelsi, A. and Baccari, B. (2006) Extended Gamma Sources Modelling Using Multipole Expansion: Application to the Tunisian Gamma Source Load Planning. Radiation Physics and Chemistry, 75, 463-472.

https://doi.org/10.1016/j.radphyschem.2005.12.024

[3] Loussaief, A. and Trabelsi, A. (2007) Dose Mapping Using Multipole Moments. Nuclear Instruments and Methods in Physics Research Section A: Accelerators, Spectrometers, Detectors and Associated Equipment, 580, 102-105. https://doi.org/10.1016/j.nima.2007.05.019

[4] Jemii, E., Mazouz, M. and Ghedira, L. (2013) Dose Rate Calculation in the Vicinity of the Tunisian Gamma Irradiation. World Journal of Nuclear Science and Technology, 3, 28-32. https://doi.org/10.4236/wjnst.2013.31005 
Submit or recommend next manuscript to OALib Journal and we will provide best service for you:

- Publication frequency: Monthly

- 9 subject areas of science, technology and medicine

- Fair and rigorous peer-review system

- Fast publication process

- Article promotion in various social networking sites (LinkedIn, Facebook, Twitter, etc.)

- Maximum dissemination of your research work

Submit Your Paper Online: Click Here to Submit

Or Contact service@oalib.com 